\title{
An algorithm for nonparametric GARCH modelling
}

\author{
Peter Bühlmann*, Alexander J. McNeil \\ Federal Institute of Technology, Department of Mathematics, ETH Zentrum, 8092 Zurich, \\ Switzerland
}

\begin{abstract}
A simple iterative algorithm for nonparametric first-order GARCH modelling is proposed. This method offers an alternative to fitting one of the many different parametric GARCH specifications that have been proposed in the literature. A theoretical justification for the algorithm is provided and examples of its application to simulated data from various stationary processes showing stochastic volatility, as well as empirical financial return data, are given. The nonparametric procedure is found to often give better estimates of the unobserved latent volatility process than parametric modelling with the standard $\operatorname{GARCH}(1,1)$ model, particularly in the presence of asymmetry and other departures from the standard GARCH specification. Extensions of the basic iterative idea to more complex time series models combining ARMA or GARCH features of possibly higher order are suggested. (C) 2002 Elsevier Science B.V. All rights reserved.
\end{abstract}

Keywords: GARCH modelling; Nonparametric methods; Volatility estimation

\section{Introduction}

Stationary time series data showing fluctuating volatility and, in particular, financial return series have provided the impetus for the study of a whole series of econometric time series models that may be grouped under the general heading of GARCH (generalised, auto-regressive, conditionally heteroscedastic models). Examples include the original ARCH model of Engle (1982), the standard GARCH model (Bollerslev, 1986), exponential GARCH or EGARCH (Nelson, 1991), integrated GARCH or IGARCH (Engle and Bollerslev, 1986), and various threshold GARCH models (Glosten et al., 1993). There are a number of useful review articles giving details of these models and their many variants; these include Bollerslev et al. (1992), Bollerslev et al. (1994) and Shephard (1996). In all of these models the hidden variable volatility depends

\footnotetext{
* Corresponding author. Tel.: +41-1-632-7338; fax: +41-1-632-1228.

E-mail addresses: buhlmann@stat.math.ethz.ch (P. Bühlmann), mcneil@math.ethz.ch (A.J. McNeil).
} 
parametrically on lagged values of the process and lagged values of volatility. The standard approach to fitting these models to data involves nonlinear maximum likelihood estimation.

We propose a first nonparametric approach to GARCH modelling which is less sensitive to model misspecification. We concentrate on a model, motivated by the natural idea in finance, where the hidden volatility depends nonparametrically on one lagged volatility and one lagged value of the process; we term this model a nonparametric GARCH $(1,1)$ process. Nonparametric estimation is a feasible alternative because, in a sense, volatility is only partially hidden. It is possible to devise an iterative scheme to estimate the volatility process and thus, perhaps surprisingly, to overcome the problem of latency. This scheme makes use of a bivariate smoother and is thus very easy to implement. Using a software package that provides bivariate smoothing such as S-Plus, we are simply required to program an additional loop.

We suggest that such an iterative bivariate smoothing scheme should have essentially the same convergence rate of estimation accuracy as one classical bivariate smoothing step, which is usually of the order $n^{-1 / 3}$ where $n$ denotes the sample size (Stone, 1982). Although the nonparametric $\operatorname{GARCH}(1,1)$-volatility process depends on the infinite past of the process, its estimation can be achieved in a manner that avoids the curse of dimensionality. This is not the case with higher-order nonparametric ARCH modelling. In contrast to the parametric case, one should not use an ARCH-approximation scheme in the nonparametric framework: an expansion of the nonparametric $\operatorname{GARCH}(1,1)$-model into a nonparametric $\mathrm{ARCH}(\infty)$-model and estimation of the latter with a high order nonparametric $\operatorname{ARCH}(p)$-model is prohibitive. The curse of dimensionality would at best lead to an estimation rate of order $n^{-2 /(4+p)}$ (see again Stone, 1982).

Another way to deal with the curse of dimensionality is to consider multiplicative $\operatorname{ARCH}(p)$-models with estimation rate usually of order $n^{-2 / 5}$ (Yang et al., 1999). But from an interpretational view, we strongly prefer the nonparametric first-order GARCH model where volatility depends only on one-lagged values of the time series and the volatility.

The basic idea of our iterative estimation algorithm allows for many extensions to other time series models with some form of latency. These extensions include nonparametric GARCH models of higher order, nonparametric ARMA models for prediction of conditional expectations and nonparametric GARCH models with a general additive structure for the conditional variance; we give examples in Section 4. To begin with we present in Section 2 the basic first-order nonparametric model and its estimation algorithm, for which we provide a justification. In Section 3 we provide examples of the use of the algorithm on both simulated and real data.

\section{The basic model}

In this paper, we consider stationary stochastic processes $\left\{X_{t} ; t \in \mathbb{Z}\right\}$ adapted to the filtration $\left\{\mathscr{F}_{t} ; t \in \mathbb{Z}\right\}$ with $\mathscr{F}_{t}=\sigma\left(\left\{X_{s} ; s \leqslant t\right\}\right)$, and having the form

$$
\begin{aligned}
X_{t} & =\sigma_{t} Z_{t}, \\
\sigma_{t}^{2} & =f\left(X_{t-1}, \sigma_{t-1}^{2}\right),
\end{aligned}
$$


where $\left\{Z_{t} ; t \in \mathbb{Z}\right\}$ is an iid innovation series with zero mean, unit variance and a finite fourth moment. $Z_{t}$ is assumed independent of $\left\{X_{s} ; s<t\right\}$, and $f: \mathbb{R} \times \mathbb{R}_{+} \rightarrow \mathbb{R}_{+}$is a strictly positive-valued function; $\sigma_{t}^{2}$ is then the conditional variance $\operatorname{Var}\left[X_{t} \mid \mathscr{F}_{t-1}\right]$ and $\sigma_{t}$ is known as the volatility.

Eqs. (1) specify a general discrete time stochastic volatility process of first order that includes the parametric $\operatorname{ARCH}(1)$ and $\operatorname{GARCH}(1,1)$ models, but which also allows for a more complicated dependence of the present volatility on the past. For example, for the parametric $\operatorname{GARCH}(1,1)$ model,

$$
f\left(x, \sigma^{2}\right)=\alpha_{0}+\alpha_{1} x^{2}+\beta \sigma^{2}, \quad \alpha_{0}, \alpha_{1}, \beta>0
$$

and for the switching volatility GARCH (SV-GARCH) model of Fornari and Mele (1997) which we will later use in a simulation study

$$
f\left(x, \sigma^{2}\right)=\alpha_{0}+\alpha_{1} x^{2}+\left(\beta_{1} \mathbf{1}_{\{x>0\}}+\beta_{2} \mathbf{1}_{\{x \leqslant 0\}}\right) \sigma^{2} .
$$

In this paper, we leave the exact form of $f$ unspecified and attempt to estimate it by nonparametric means.

To this end we observe first that the model (1) can be written in terms of an additive noise as follows:

$$
\begin{aligned}
& X_{t}^{2}=f\left(X_{t-1}, \sigma_{t-1}^{2}\right)+V_{t}, \\
& V_{t}=f\left(X_{t-1}, \sigma_{t-1}^{2}\right)\left(Z_{t}^{2}-1\right),
\end{aligned}
$$

where $V_{t}$ is a martingale difference series with $\mathbb{E}\left[V_{t}\right]=\mathbb{E}\left[V_{t} \mid \mathscr{F}_{t-1}\right]=0$ and $\operatorname{Cov}\left[V_{s}, V_{t}\right]=$ $\operatorname{Cov}\left[V_{s}, V_{t} \mid \mathscr{F}_{t-1}\right]=0$ for $s<t$. It follows that

$$
\mathbb{E}\left[X_{t}^{2} \mid \mathscr{F}_{t-1}\right]=f\left(X_{t-1}, \sigma_{t-1}^{2}\right)
$$

and

$$
\operatorname{Var}\left[X_{t}^{2} \mid \mathscr{F}_{t-1}\right]=f^{2}\left(X_{t-1}, \sigma_{t-1}^{2}\right)\left(\mathbb{E}\left[Z_{t}^{4}\right]-1\right) .
$$

This suggests we could estimate $f$ by regressing $X_{t}^{2}$ on the lagged variables $X_{t-1}$ and $\sigma_{t-1}^{2}$ using a nonparametric smoothing technique. The conditional heteroscedasticity of the series $X_{t}^{2}$ suggests a weighted regression might be used. The principal problem is that the volatility $\sigma_{t-1}$ is an unobserved latent variable. This problem is overcome in the following algorithm.

\subsection{An estimation algorithm}

Assume we have a data sample $\left\{X_{t} ; 1 \leqslant t \leqslant n\right\}$, ideally from a process satisfying (1).

(1) Calculate a first estimate of volatility $\left\{\hat{\sigma}_{t, 0} ; 1 \leqslant t \leqslant n\right\}$ by fitting an ordinary parametric $\operatorname{GARCH}(1,1)$ model by standard maximum likelihood. Set $m=1$.

(2) Regress $\left\{X_{t}^{2} ; 2 \leqslant t \leqslant n\right\}$ against $\left\{X_{t-1} ; 2 \leqslant t \leqslant n\right\}$ and $\left\{\hat{\sigma}_{t-1, m-1}^{2} ; 2 \leqslant t \leqslant n\right\}$ using a nonparametric smoothing procedure to obtain an estimate $\hat{f}_{m}$ of $f$. As an added refinement a weighted regression can be performed with regression weights $\left\{\hat{\sigma}_{t, m-1}^{-2} ; 2 \leqslant t \leqslant n\right\}$; our experience with simulations suggests this mostly yields 
slightly improved estimates. (We thereby assume that $\mathbb{E}\left[Z_{t}^{4}\right]<\infty$ and that $f\left(x, \sigma^{2}\right)$ is finite for all $x, \sigma^{2}$, which implies the existence of $\left.\operatorname{Var}\left(X_{t}^{2} \mid \mathscr{F}_{t-1}\right)\right)$.

(3) Calculate $\left\{\hat{\sigma}_{t, m}^{2}=\hat{f}_{m}\left(X_{t-1}, \hat{\sigma}_{t-1, m-1}^{2}\right) ; 2 \leqslant t \leqslant n\right\}$ and impute some sensible value for $\hat{\sigma}_{1, m}^{2}$, for example $\hat{\sigma}_{1, m-1}^{2}$.

(4) Increment $m$ and return to step two if $m<M$, where $M$ is a prespecified maximum number of iterations.

Our experience, gathered from a number of simulation experiments, suggests that if the nonparametric estimation steps will provide an improvement over the parametric GARCH estimation of volatility, then this improvement will be attained in a small number of steps 1-4. Thereafter, there is little to pick and choose between volatility estimates $\hat{\sigma}_{t, m}$ for various values of $m$. The algorithm can however often be improved by averaging over the final $K$ such estimates to obtain

$$
\hat{\sigma}_{t, *}=(1 / K) \sum_{m=M-K+1}^{M} \hat{\sigma}_{t, m}
$$

and then performing a final regression of $X_{t}^{2}$ against $X_{t-1}$ and $\hat{\sigma}_{t-1, *}^{2}$ to get final estimates $\hat{f}$ of $f$ and $\hat{\sigma}_{t}^{2}=\hat{f}\left(X_{t-1}, \hat{\sigma}_{t-1, *}^{2}\right)$; we refer to this as a final smoothing step. We average over volatilities rather than squared volatilities since the estimation of volatility is our main goal.

Note that positivity of the estimate $\hat{\sigma}_{t}^{2}$ is guaranteed when using the algorithm with a smoothing technique of the form $\sum_{t} w_{t} X_{t}^{2}$ with positive weights $w_{t}$ summing to one, for example kernel smoothing with a positive kernel.

The parameters of the estimation algorithm are $n$, the sample size; $M$, the number of basic iterations and $K$, the number of estimates used in the final smoothing step. There will also be a bandwidth parameter to choose in the nonparametric regression method.

\subsection{Justification of the algorithm}

The following assumption is crucial for our justification of consistency of the estimation algorithm.

Assumption A1 (contraction with respect to hidden variable).

$$
\sup _{x \in \mathbb{R}}\left|f\left(x, \sigma^{2}\right)-f\left(x, \tau^{2}\right)\right| \leqslant D\left|\sigma^{2}-\tau^{2}\right| \text { for some } 0<D<1 \text {, for all } \sigma^{2}, \tau^{2} \in \mathbb{R}_{+} .
$$

Assumption A1 is crucial in our reasoning and we conjecture that without a suitable contraction property of the function $f$ with respect to the second, hidden argument there is no hope of getting a consistent estimate with our iterative scheme. The condition holds for stationary parametric $\operatorname{GARCH}(1,1)$ models and also for the asymmetric models A and B in Section 3.1.

The population case: A key feature of the algorithm can be understood when simplifying to the case of no estimation error. We recursively define

$$
\begin{aligned}
& f_{t, m}\left(x, \sigma^{2}\right)=\mathbb{E}\left[X_{t}^{2} \mid X_{t-1}=x, \sigma_{t-1, m-1}^{2}=\sigma^{2}\right], \\
& \sigma_{t, m}^{2}=f_{t, m}\left(X_{t-1}, \sigma_{t-1, m-1}^{2}\right), m=1,2, \ldots ; t \in \mathbb{Z},
\end{aligned}
$$


where $\sigma_{t, 0}^{2}$ are some starting values assumed to be elements of $\mathscr{F}_{t-1}$ for all $t \in \mathbb{Z}$, i.e. they are independent from $\left\{Z_{s} ; s \geqslant t\right\}$. The $\sigma_{t, m}^{2}$ are the population quantities corresponding to the estimates $\hat{\sigma}_{t, m}^{2}$ of the algorithm. They can be thought of as representing the case with infinitely many observations where estimation errors would be zero.

Assumption A2 (moment assumption).

$$
C_{1}:=\mathbb{E}\left|\sigma_{t}\right|^{4}<\infty, \quad C_{2}:=\mathbb{E}\left|\sigma_{t, 0}\right|^{4}<\infty .
$$

Theorem 1. Assume that $\left\{X_{t} ; t \in \mathbb{Z}\right\}$ is as in (1), satisfying assumptions $A 1$ and $A 2$. Denote by $\|\cdot\|_{2}$ the $L_{2}$-norm, i.e. $\|Y\|_{2}=\left(\mathbb{E}|Y|^{2}\right)^{1 / 2}$. Then, in the mth iteration step

$$
\left\|\sigma_{t, m}^{2}-\sigma_{t}^{2}\right\|_{2} \leqslant D^{m}\left(C_{1}+C_{2}\right), \quad t=m+2, m+3, \ldots .
$$

A proof is given in the appendix. Theorem 1 shows that in the case of no estimation error, iteration from arbitrary starting values $\sigma_{t, 0}^{2}(t \in \mathbb{Z})$ leads exponentially fast to the true squared volatility $\sigma_{t}^{2}$, due to the contraction property in assumption A1. It thus indicates the feasibility of solving the problem of nonparametric GARCH estimation with a simple iterative smoothing scheme.

An approach dealing with estimation errors: A rigorous mathematical analysis about the estimation error in our procedure seems very difficult, due to the iterative structure of the algorithm. Although Theorem 2 below is mathematically rigorous, its assumptions are hard to justify. Nevertheless, we believe that the theoretical approach presented here gives some useful insight.

We bound the quantity of interest as

$$
\left\|\hat{\sigma}_{t, m}^{2}-\sigma_{t}^{2}\right\|_{2} \leqslant\left\|\hat{\sigma}_{t, m}^{2}-\tilde{\sigma}_{t, m}^{2}\right\|_{2}+\left\|\tilde{\sigma}_{t, m}^{2}-\sigma_{t, m}^{2}\right\|_{2}+\left\|\sigma_{t, m}^{2}-\sigma_{t}^{2}\right\|_{2} .
$$

Thereby,

$$
\begin{aligned}
& \tilde{f}_{t, m}\left(x, \sigma^{2}\right)=\mathbb{E}\left[X_{t}^{2} \mid X_{t-1}=x, \hat{\sigma}_{t-1, m-1}^{2}=\sigma^{2}\right], \\
& \tilde{\sigma}_{t, m}^{2}=\tilde{f}_{t, m}\left(X_{t-1}, \hat{\sigma}_{t-1, m-1}^{2}\right),
\end{aligned}
$$

the latter being a true conditional expectation as a function of $X_{t-1}$ and the estimate $\hat{\sigma}_{t-1, m-1}^{2} . \tilde{\sigma}_{t, m}^{2}$ is an intermediate term between the population quantity $\sigma_{t, m}^{2}$ above and the estimate $\hat{\sigma}_{t, m}^{2}$. The terms on the right-hand side in (2) can be controlled as follows. The third one is bounded by Theorem 1; the first and second are controlled using Assumptions A3 and A4 below.

\section{Assumption A3.}

$$
\begin{aligned}
& \mathbb{E}\left[\left\{\tilde{\sigma}_{t, m}^{2}-\sigma_{t, m}^{2}\right\}^{2}\right]=\mathbb{E}\left[\left\{\tilde{f}_{t, m}\left(X_{t-1}, \hat{\sigma}_{t-1, m-1}^{2}\right)-f_{t, m}\left(X_{t-1}, \sigma_{t-1, m-1}^{2}\right)\right\}^{2}\right] \\
& \quad \leqslant G^{2} \mathbb{E}\left[\left\{\hat{\sigma}_{t-1, m-1}^{2}-\sigma_{t-1, m-1}^{2}\right\}^{2}\right] \quad \text { for some } 0<G<1
\end{aligned}
$$

for $t=m+2, m+3, \ldots$ and $m=1,2, \ldots$

Assumption A4 ( $\mathscr{L}_{2}$ estimation error).

$$
\Delta_{n}^{\left(\mathscr{L}_{2}\right)}=\sup _{m \geqslant 1} \max _{m+2 \leqslant t \leqslant n}\left\|\hat{\sigma}_{t, m}^{2}-\tilde{\sigma}_{t, m}^{2}\right\|_{2} \rightarrow 0 \quad \text { as } n \rightarrow \infty .
$$


Theorem 2. Assume that $\left\{X_{t} ; t \in \mathbb{Z}\right\}_{t}$ is as in (1), satisfying assumptions $A 1-A 4$ and $\max _{2 \leqslant t \leqslant n} \mathbb{E}\left|\hat{\sigma}_{t, 0}^{2}\right|^{4} \leqslant C_{3}<\infty$ for all $n$. Denote by $\|\cdot\|_{2}$ the $L_{2}$-norm, i.e. $\|Y\|_{2}=$ $\left(\mathbb{E}|Y|^{2}\right)^{1 / 2}$. Then, the estimator $\hat{\sigma}_{t, m}^{2}$ in the $m$ th step of the algorithm satisfies

$$
\begin{aligned}
& \left\|\hat{\sigma}_{t, m}^{2}-\sigma_{t}^{2}\right\|_{2} \leqslant \Delta_{n}^{\left(\mathscr{L}_{2}\right)} /(1-G)+G^{m}\left(C_{1}+C_{3}\right)+D^{m}\left(C_{1}+C_{2}\right) \\
& \quad \text { for all } t=m+2, m+3, \ldots, n .
\end{aligned}
$$

Particularly, by choosing $m_{n}=C\left\{-\log \left(\Delta_{n}^{\left(\mathscr{L}_{2}\right)}\right)\right\}$ for some $C$ satisfying $C \geqslant \max \{-1 /$ $\log (D),-1 / \log (G)\}$, we have

$$
\max _{m_{n}+2 \leqslant t \leqslant n}\left\|\hat{\sigma}_{t, m_{n}}^{2}-\sigma_{t}^{2}\right\|_{2}=\mathrm{O}\left(\Delta_{n}^{\left(\mathscr{L}_{2}\right)}\right)=o(1) \quad \text { as } n \rightarrow \infty .
$$

A proof is given in the appendix. Note that similar asymptotic results also hold for the final smoothed estimate $\hat{\sigma}_{t}^{2}$.

The last statement of Theorem 2 says that the accuracy of the estimation algorithm is of the same order as the maximal error $\Delta_{n}^{\left(\mathscr{L}_{2}\right)}$ in one smoothing step. Since the smoothing problem is bivariate, we expect $\Delta_{n}^{\left(\mathscr{L}_{2}\right)}=\mathrm{O}\left(n^{-1 / 3}\right)$ (or slightly slower) with a second-order kernel, provided some appropriate smoothness conditions on the function $f$ hold and rate-optimal bandwidth choice is used (Stone, 1982), although the volatility of the nonparametric GARCH model at time $t$ is a function of the infinite past $X_{t-1}, X_{t-2}, \ldots$.

We end this section with a brief discussion of Assumptions A3 and A4, which are needed exclusively to deal with the case of estimation error.

Assumption A3 can be viewed as a requirement on projections: $\mathbb{E}\left[\left\{\tilde{\sigma}_{t, m}^{2}-\sigma_{t, m}^{2}\right\}^{2}\right]=$ $\left(\mathbb{E}\left[X_{t}^{2} \mid X_{t-1}, \hat{\sigma}_{t-1, m-1}^{2}\right]-\mathbb{E}\left[X_{t}^{2} \mid X_{t-1}, \sigma_{t-1, m-1}^{2}\right]\right)^{2}$. The requirement in A3 is thus a contraction for the difference of projections (conditional expectations) with respect to $\mathbb{E}\left[\left\{\hat{\sigma}_{t-1, m-1}^{2}-\sigma_{t-1, m-1}^{2}\right\}^{2}\right]$, an expected difference between the variables causing the projections to be distinct. Assumption A4 controls the $\mathscr{L}_{2}$ estimation error when nonparametrically regressing $X_{t}^{2}$ versus $X_{t-1}$ and $\hat{\sigma}_{t-1, m-1}^{2}$. A rigorous justification for appropriateness of our Assumptions A3 and A4 is not given here and still an open area for future research in theoretical statistics.

\section{Illustrative examples}

\subsection{Simulation experiments}

The aim of these simulation experiments is to develop some feeling for the kinds of processes where the nonparametric procedure can offer better estimates of the unobserved volatility than standard parametric GARCH modelling. The simple GARCH( 1,1$)$ model might be considered a natural starting point in any modelling exercise with real data where we have no strong prior information that would lead us to choose a more sophisticated parametric specification. 


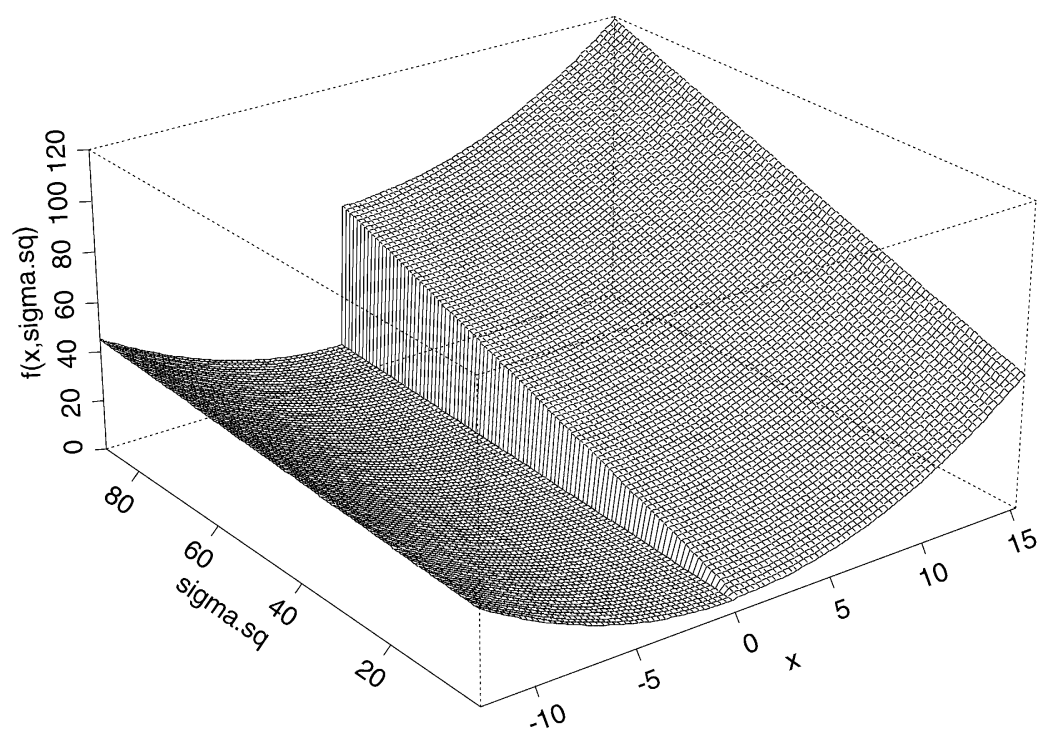

Fig. 1. Simulation experiment. The volatility surface $f\left(x, \sigma^{2}\right)=5+0.2 x^{2}+\left(0.751_{\{x>0\}}+0.11_{\{x \leqslant 0\}}\right) \sigma^{2}$; note the asymmetry depending on the sign of $x$.

The processes we consider have the following three volatility surfaces, all of which conform to (1).

$$
\begin{aligned}
\mathrm{A}: f\left(x, \sigma^{2}\right)= & 5+0.2 x^{2}+\left(0.75 \cdot \mathbf{1}_{\{x>0\}}+0.1 \cdot \mathbf{1}_{\{x \leqslant 0\}}\right) \sigma^{2} . \\
\mathrm{B}: f\left(x, \sigma^{2}\right)= & 8+\left(0.001 \cdot \mathbf{1}_{\{x>0\}}+0.45 \cdot \mathbf{1}_{\{x \leqslant 0\}}\right) x^{2}+0.5 \sigma^{2} . \\
\mathrm{C}: f\left(x, \sigma^{2}\right)= & (1-\exp (-0.2|x \| \sigma|))\left(5+0.2 x^{2}+\left(0.75 \cdot \mathbf{1}_{\{x>0\}}\right.\right. \\
& \left.\left.+0.1 \cdot \mathbf{1}_{\{x \leqslant 0\}}\right) \sigma^{2}\right)^{3 / 4} .
\end{aligned}
$$

Thus the first two processes (A and B) are threshold GARCH processes where switching asymmetry has been built into either the ARCH or GARCH effect, along the lines of models suggested by Glosten et al. (1993) and Fornari and Mele (1997). The volatility surface for process A is depicted in Fig. 1. Clearly if we were to correctly deduce the form of the underlying model for these data and fit this model parametrically we would get good results. However, the third process $\mathrm{C}$ is a more exotic process, with threshold effects as well as a damping term, where it is unlikely that we would guess the correct functional relationship.

We work with realisations of length $n=1000$ points. In all experiments the number of basic iterations is set to be $M=8$ and a final smooth based on $K=5$ final iterations is performed. We use the default value of the smoothing parameter in the S-Plus implementation of a bivariate loess smoother. 

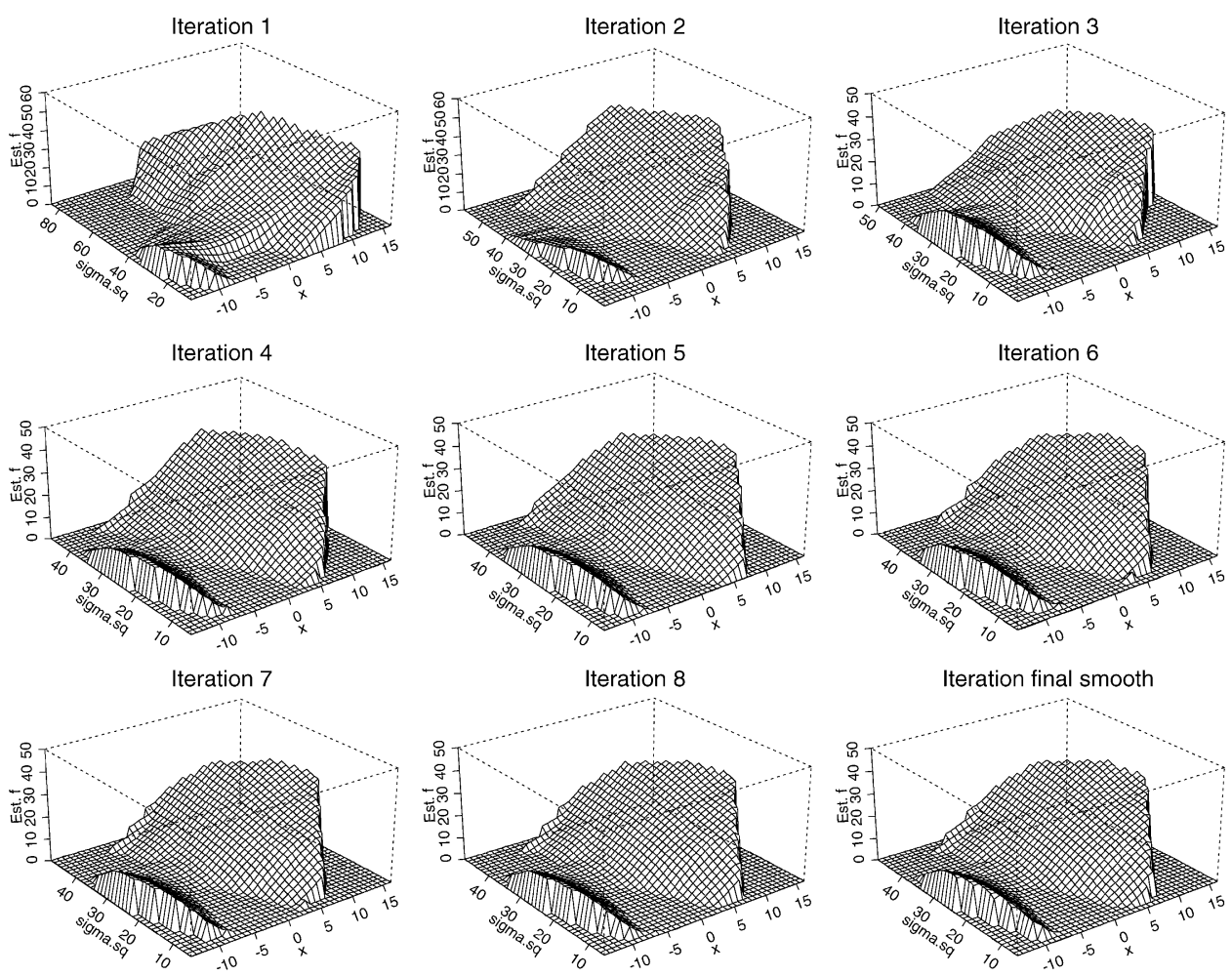

Fig. 2. Simulation experiment. The estimated surfaces $\hat{f}_{m}$ calculated at the first 8 iterations and the surface $\hat{f}$ estimated at the final smoothing stage.

In Fig. 2 we show typical results for one realisation from process A. The estimated surfaces $\hat{f}_{m}$ calculated at the first 8 iterations and the surface calculated at the final smoothing stage are shown. Comparison with Fig. 1 indicates that the nonparametric algorithm is recovering the essential features of the volatility surface. This series of nine pictures provides some graphical reassurance that the method is converging and illustrates the first envisaged use of the algorithm, which is as an exploratory graphical tool for investigating the dependence of the present volatility on the immediate past.

For the same realisation we compare in Fig. 3 the volatility estimates we obtain with the true volatility trajectory for an arbitrarily selected section of 70 observations. In the left-hand picture it is clear that the parametric estimate derived from $\operatorname{GARCH}(1,1)$ modelling is unable to follow the true volatility closely through its peaks and troughs; on the other hand, in the right-hand picture we see that the nonparametric GARCH estimate derived from the final smoothing round is fairly faithful to the true volatility.

To provide a quantitative assessment of the accuracy of the volatility estimates we calculate both an average squared estimation error and an average absolute estimation error for each realisation at each iteration of the method. Because estimates of volatility at the first few time points may be unreliable we generally omit $r=10$ values from 

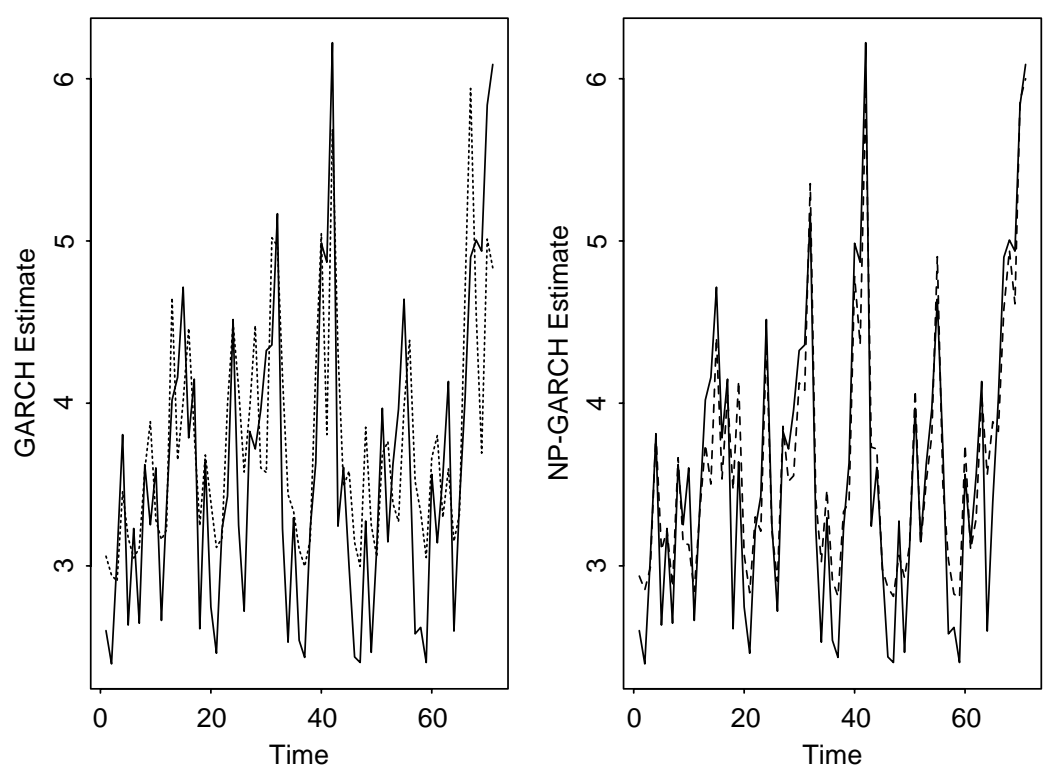

Fig. 3. Simulation experiment. For an arbitrary section of 70 observations from a simulated realisation of Process A, the left-hand plot shows true volatility (solid line) compared with a parametric $\operatorname{GARCH}(1,1)$ estimate (dotted line) and the right-hand plot shows true volatility compared with the nonparametric GARCH estimate obtained after a final smooth (dashed line).

this calculation. Our measures of estimation error for a single realisation at iteration $m$ are

$$
\operatorname{SE}\left(\hat{\sigma}_{,, m}\right)=\frac{1}{n-r} \sum_{t=r+1}^{n}\left(\hat{\sigma}_{t, m}-\sigma_{t}\right)^{2}, \quad \operatorname{AE}\left(\hat{\sigma}_{\cdot, m}\right)=\frac{1}{n-r} \sum_{t=r+1}^{n}\left|\hat{\sigma}_{t, m}-\sigma_{t}\right| .
$$

For each process we generate 50 independent realisations and average our volatility estimation error statistics to provide an estimate of mean squared error (MSE) and mean absolute error (MAE); we also use the replications to give standard errors for our MSE and MAE estimates.

Results for data generated with normal innovations are presented in Table 1 and Table 2; results for scaled $t$ innovations with 8 degrees of freedom are presented in Tables 3 and 4 . In the case of normal innovations we also show a direct comparison of the method incorporating weighting with the unweighted method. Although both methods offer an improvement over ordinary $\operatorname{GARCH}(1,1)$ modelling it is clear from the figures in the table that the weighted procedure should be preferred to the unweighted procedure. For $t$ innovations this finding also holds and we present results for the weighted procedure only.

In Tables 1 and 2 we see that in all cases 3 or 4 iterations of the nonparametric method are sufficient to obtain a substantial improvement on the parametric estimates, and the volatility estimate obtained at the final smoothing step is mostly the best estimate of all. Concentrating on the weighted procedure and this final smoothing step 
Table 1

Estimated mean squared volatility estimation error (MSE) and mean absolute volatility estimation error (MAE) at each iteration of the method for random samples of $n=1000$ points from the processes A and $\mathrm{B}$, in the case of Gaussian innovations

\begin{tabular}{|c|c|c|c|c|c|c|c|c|}
\hline \multirow{2}{*}{$\begin{array}{l}\text { Iter. } \\
(m)\end{array}$} & \multicolumn{2}{|c|}{ Weighted } & \multicolumn{2}{|c|}{ Unweighted } & \multicolumn{2}{|c|}{ Weighted } & \multicolumn{2}{|c|}{ Unweighted } \\
\hline & MSE & Std. err. & MSE & Std. err. & MAE & Std. err. & MAE & Std. err. \\
\hline \multicolumn{9}{|c|}{$\mathrm{A}: f\left(x, \sigma^{2}\right)=5+0.2 x^{2}+\left(0.75 \cdot \mathbf{1}_{\{x>0\}}+0.1 \cdot \mathbf{1}_{\{x \leqslant 0\}}\right) \sigma^{2}$} \\
\hline 0 & 0.47 & 0.008 & 0.47 & 0.008 & 0.57 & 0.004 & 0.57 & 0.004 \\
\hline 1 & 0.34 & 0.012 & 0.37 & 0.016 & 0.41 & 0.006 & 0.42 & 0.007 \\
\hline 2 & 0.25 & 0.009 & 0.26 & 0.012 & 0.35 & 0.005 & 0.36 & 0.007 \\
\hline 3 & 0.25 & 0.012 & 0.26 & 0.014 & 0.34 & 0.007 & 0.35 & 0.007 \\
\hline 4 & 0.22 & 0.007 & 0.25 & 0.013 & 0.33 & 0.005 & 0.35 & 0.007 \\
\hline 5 & 0.22 & 0.008 & 0.25 & 0.013 & 0.33 & 0.005 & 0.35 & 0.007 \\
\hline 6 & 0.22 & 0.009 & 0.24 & 0.010 & 0.33 & 0.006 & 0.34 & 0.006 \\
\hline 7 & 0.22 & 0.009 & 0.25 & 0.013 & 0.33 & 0.006 & 0.35 & 0.007 \\
\hline 8 & 0.22 & 0.009 & 0.24 & 0.012 & 0.33 & 0.006 & 0.35 & 0.007 \\
\hline$*$ & 0.22 & 0.008 & 0.25 & 0.012 & 0.33 & 0.006 & 0.35 & 0.007 \\
\hline \multicolumn{9}{|c|}{$\mathrm{B}: f\left(x, \sigma^{2}\right)=8+\left(0.001 \cdot \mathbf{1}_{\{x>0\}}+0.45 \cdot \mathbf{1}_{\{x \leqslant 0\}}\right) x^{2}+0.5 \sigma^{2}$} \\
\hline 0 & 1.02 & 0.029 & 1.02 & 0.029 & 0.67 & 0.007 & 0.67 & 0.007 \\
\hline 1 & 1.10 & 0.185 & 1.90 & 0.658 & 0.59 & 0.032 & 0.72 & 0.091 \\
\hline 2 & 0.67 & 0.115 & 0.75 & 0.121 & 0.47 & 0.024 & 0.50 & 0.027 \\
\hline 3 & 0.81 & 0.271 & 0.92 & 0.162 & 0.47 & 0.052 & 0.53 & 0.039 \\
\hline 4 & 0.63 & 0.110 & 0.69 & 0.123 & 0.43 & 0.024 & 0.45 & 0.023 \\
\hline 5 & 0.60 & 0.090 & 1.01 & 0.193 & 0.43 & 0.021 & 0.54 & 0.045 \\
\hline 6 & 0.53 & 0.067 & 1.14 & 0.521 & 0.41 & 0.017 & 0.53 & 0.078 \\
\hline 7 & 0.59 & 0.080 & 1.04 & 0.263 & 0.43 & 0.022 & 0.53 & 0.053 \\
\hline 8 & 0.50 & 0.051 & 0.67 & 0.109 & 0.40 & 0.015 & 0.46 & 0.029 \\
\hline$*$ & 0.47 & 0.047 & 0.86 & 0.177 & 0.39 & 0.014 & 0.50 & 0.041 \\
\hline
\end{tabular}

See Table 2 for process C. Iteration 0 corresponds to parametric GARCH; iteration marked $*$ is the final smoothing step. Estimates of MSE, MAE, and their standard errors (std. err.) are based on 50 replications of the simulation experiment. The method incorporating weighting is also compared with the unweighted method.

we find that the MSEs are reduced by $53 \%, 54 \%$ and $40 \%$ for processes A, B and C respectively. The MAEs are reduced by $42 \%, 42 \%$ and $32 \%$.

The results in Tables 3 and 4 for scaled $t$ innovations with 8 degrees of freedom also show that the nonparametric procedure can improve on the volatility estimates delivered by the $\operatorname{GARCH}(1,1)$ process. In this case the improvements are $35 \%, 28 \%$ and $27 \%$ for the MSEs, and $34 \%, 28 \%$ and $21 \%$ for the MAEs.

Our experience gained over a number of simulation experiments is that the volatility surface must deviate fairly clearly from that of the $\operatorname{GARCH}(1,1)$ process before these kinds of improvement are registered. For simple additive surfaces of the form

$$
f\left(x, \sigma^{2}\right)=g(x)+h\left(\sigma^{2}\right)
$$

where $g: \mathbb{R} \rightarrow \mathbb{R}_{+}$is a positive-valued function satisfying $g(x)=g(-x)$ (such as $g(x)=\alpha|x|, 0<\alpha<1)$ and $h: \mathbb{R}_{+} \rightarrow \mathbb{R}_{+}$is a positive-valued nondecreasing function (such as $h\left(\sigma^{2}\right)=\beta \sigma, 0<\beta<1$ ), the performance of ordinary $\operatorname{GARCH}(1,1)$ is often 
Table 2

Estimated mean squared volatility estimation error (MSE) and mean absolute volatility estimation error (MAE) at each iteration of the method for random samples of $n=1000$ points from process $\mathrm{C}$, in the case of Gaussian innovations

\begin{tabular}{|c|c|c|c|c|c|c|c|c|}
\hline \multirow{2}{*}{$\begin{array}{l}\text { Iter. } \\
(m)\end{array}$} & \multicolumn{2}{|c|}{ Weighted } & \multicolumn{2}{|c|}{ Unweighted } & \multicolumn{2}{|c|}{ Weighted } & \multicolumn{2}{|c|}{ Unweighted } \\
\hline & MSE & Std. err. & MSE & Std. err. & MAE & Std. err. & MAE & Std. err. \\
\hline \multicolumn{9}{|c|}{$\mathrm{C}: f\left(x, \sigma^{2}\right)=\left(1-\mathrm{e}^{-0.2|x \| \sigma|}\right)\left(5+0.2 x^{2}+\left(0.75 \cdot \mathbf{1}_{\{x>0\}}+0.1 \cdot \mathbf{1}_{\{x \leqslant 0\}}\right) \sigma^{2}\right)^{3 / 4}$} \\
\hline 0 & 0.112 & 0.002 & 0.112 & 0.002 & 0.28 & 0.002 & 0.28 & 0.002 \\
\hline 1 & 0.073 & 0.003 & 0.073 & 0.003 & 0.20 & 0.004 & 0.20 & 0.004 \\
\hline 2 & 0.070 & 0.003 & 0.071 & 0.003 & 0.20 & 0.004 & 0.20 & 0.004 \\
\hline 3 & 0.070 & 0.003 & 0.072 & 0.003 & 0.20 & 0.004 & 0.20 & 0.004 \\
\hline 4 & 0.067 & 0.003 & 0.070 & 0.003 & 0.19 & 0.004 & 0.20 & 0.004 \\
\hline 5 & 0.069 & 0.003 & 0.070 & 0.002 & 0.19 & 0.004 & 0.20 & 0.004 \\
\hline 6 & 0.069 & 0.003 & 0.070 & 0.003 & 0.19 & 0.004 & 0.20 & 0.004 \\
\hline 7 & 0.067 & 0.003 & 0.068 & 0.002 & 0.19 & 0.004 & 0.20 & 0.004 \\
\hline 8 & 0.068 & 0.002 & 0.069 & 0.003 & 0.19 & 0.004 & 0.20 & 0.004 \\
\hline$*$ & 0.067 & 0.003 & 0.069 & 0.003 & 0.19 & 0.004 & 0.20 & 0.004 \\
\hline
\end{tabular}

See Table 1 for further details.

comparable to that of the nonparametric procedure, even though $\operatorname{GARCH}(1,1)$ assumes an erroneous parametric form. For complicated additive functions we note that our procedure can be adapted to fit general nonparametric additive models; for an outline see Section 4.2. When the true process is exactly $\operatorname{GARCH}(1,1)$ then we would expect the parametric procedure to outperform the nonparametric one, and it does so.

\subsection{Empirical example}

For an example with real data we take a 1000-day excerpt from the time series of daily percentage returns on the BMW share price (see Fig. 4). Our time series runs from the 2nd June 1986 to the 30th March 1990 and contains the period of high volatility around the 1987 stock market crash.

In Fig. 5 we show again the graphical output of the algorithm for the first 8 iterations and the final smoothing step. The pictures have been rotated so that we view them along the line $x=0$. These estimated volatility surfaces show some evidence of an asymmetric effect, which depends on the sign of the last observation; as long as volatility is low, a large positive return appears to have a much more modest effect on the next day's volatility than does a large negative return. This asymmetric effect of new information is a well-known phenomenon in financial time series and our method clearly picks it up. Finally in Fig. 6 we show the estimated volatilities derived from parametric modelling and the final-smooth stage of nonparametric modelling.

For comparison, we also fitted two parametric, first-order, threshold GARCH models conforming to (1). The first model M1 is a four-parameter model of the type used by Glosten et al. (1993) (i.e. Process B of the previous section); the second model M2 is a generalised six-parameter model incorporating threshold effects for the ARCH and GARCH terms, as well as the constant parameter. The conditional variances are given 
Table 3

Estimated mean squared volatility estimation error (MSE) and mean absolute volatility estimation error (MAE) at each iteration of the weighted method for random samples of $n=1000$ points from the processes A and $\mathrm{B}$, in the case of $t$ innovations with 8 degrees of freedom

\begin{tabular}{lllll}
\hline$m$ & MSE & Std. err. & MAE & Std. err. \\
\hline Process A & & & & \\
0 & 0.52 & 0.015 & 0.59 & 0.006 \\
1 & 0.52 & 0.032 & 0.48 & 0.011 \\
2 & 0.42 & 0.050 & 0.43 & 0.018 \\
3 & 0.42 & 0.058 & 0.42 & 0.019 \\
4 & 0.42 & 0.069 & 0.42 & 0.023 \\
5 & 0.39 & 0.048 & 0.41 & 0.016 \\
6 & 0.38 & 0.041 & 0.41 & 0.015 \\
7 & 0.39 & 0.041 & 0.41 & 0.013 \\
8 & 0.37 & 0.048 & 0.40 & 0.018 \\
$*$ & 0.34 & 0.029 & 0.39 & 0.010 \\
& & & & \\
Process B & & & & 0.007 \\
0 & 1.18 & 0.052 & 0.67 & 0.025 \\
1 & 1.38 & 0.165 & 0.64 & 0.024 \\
2 & 0.94 & 0.109 & 0.54 & 0.045 \\
3 & 1.07 & 0.183 & 0.55 & 0.031 \\
4 & 0.98 & 0.165 & 0.51 & 0.028 \\
5 & 0.86 & 0.110 & 0.51 & 0.040 \\
6 & 0.98 & 0.230 & 0.51 & 0.027 \\
7 & 0.90 & 0.138 & 0.49 & 0.020 \\
8 & 0.76 & 0.078 & 0.47 & 0.029 \\
$*$ & 0.85 & 0.163 & 0.48 & \\
\hline & & & & \\
\hline
\end{tabular}

See Table 4 for process C. Iteration 0 corresponds to parametric GARCH using pseudo maximum likelihood; iteration $*$ is the final smoothing step. Estimates of MSE, MAE, and their standard errors (std. err.) are based on 50 replications of the simulation experiment.

by

$$
\begin{aligned}
& \text { M1: } \sigma_{t}^{2}=\alpha_{0}+\alpha_{1} X_{t-1}^{2} \mathbf{1}_{\left\{X_{t-1} \leqslant 0\right\}}+\alpha_{2} X_{t-1}^{2} \mathbf{1}_{\left\{X_{t-1}>0\right\}}+\beta \sigma_{t-1}^{2}, \\
& \text { M2: } \sigma_{t}^{2}=\left(\alpha_{0}+\alpha_{1} X_{t-1}^{2}+\beta_{1} \sigma_{t-1}^{2}\right) \mathbf{1}_{\left\{X_{t-1} \leqslant 0\right\}}+\left(\alpha_{2}+\alpha_{3} X_{t-1}^{2}+\beta_{2} \sigma_{t-1}^{2}\right) \mathbf{1}_{\left\{X_{t-1}>0\right\}},
\end{aligned}
$$

where all parameters $\alpha_{i}, \beta_{i}$ are positive and innovations are assumed to be standard normal. The classical $\operatorname{GARCH}(1,1)$ model with standard Gaussian innovations is also considered as the starting fit of our nonparametric iterative GARCH method. Since $\sigma_{t}$ is unobserved we cannot use squared and absolute error statistics as before and so we therefore consider the following goodness of fit measures:

$$
\begin{aligned}
& L_{2}: \frac{1}{990} \sum_{t=11}^{1000}\left(\hat{\sigma}_{t}^{2}-X_{t}^{2}\right)^{2}, \\
& -\mathrm{LL}:-\sum_{t=11}^{1000}-\log \left(\hat{\sigma}_{t}^{-1} \varphi\left(\frac{X_{t}}{\hat{\sigma}_{t}}\right)\right),
\end{aligned}
$$


Table 4

Estimated mean squared volatility estimation error (MSE) and mean absolute volatility estimation error (MAE) at each iteration of the method for random samples of $n=1000$ points from process $\mathrm{C}$, in the case of $t$ innovations with 8 degrees of freedom

\begin{tabular}{lllll}
\hline$m$ & MSE & Std. err. & MAE & Std. err. \\
\hline Process C & & & & \\
0 & 0.127 & 0.003 & 0.29 & 0.002 \\
1 & 0.108 & 0.006 & 0.24 & 0.007 \\
2 & 0.095 & 0.004 & 0.22 & 0.005 \\
3 & 0.096 & 0.005 & 0.23 & 0.006 \\
4 & 0.092 & 0.004 & 0.22 & 0.005 \\
5 & 0.093 & 0.004 & 0.22 & 0.005 \\
6 & 0.089 & 0.004 & 0.22 & 0.005 \\
7 & 0.092 & 0.004 & 0.22 & 0.005 \\
8 & 0.096 & 0.004 & 0.23 & 0.005 \\
$*$ & 0.093 & 0.004 & 0.23 & 0.005 \\
\hline
\end{tabular}

See Table 3 for further details.

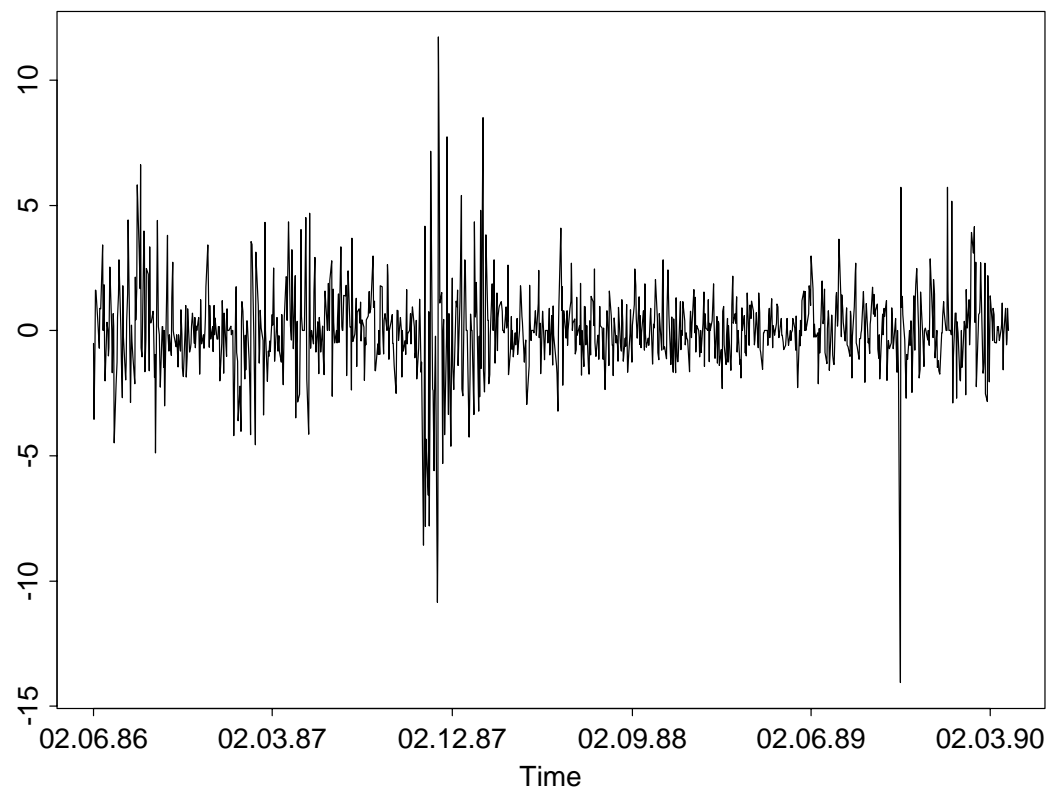

Fig. 4. Real data. A 1000-day excerpt from the time series of daily returns on the BMW share price.

$$
\text { AIC }:-2 \mathrm{LL}+2 \cdot \text { number of parameters. }
$$

The measure $L_{2}$ is a quadratic deviance measure, which is based on the additive representation of the squared series as $X_{t}^{2}=\sigma_{t}^{2}+V_{t}$, where $V_{t}$ is a martingale difference series with mean zero (see Section 2). The statistic - LL is the negative log-likelihood and AIC is a penalised negative log-likelihood, designed to measure the outsample 

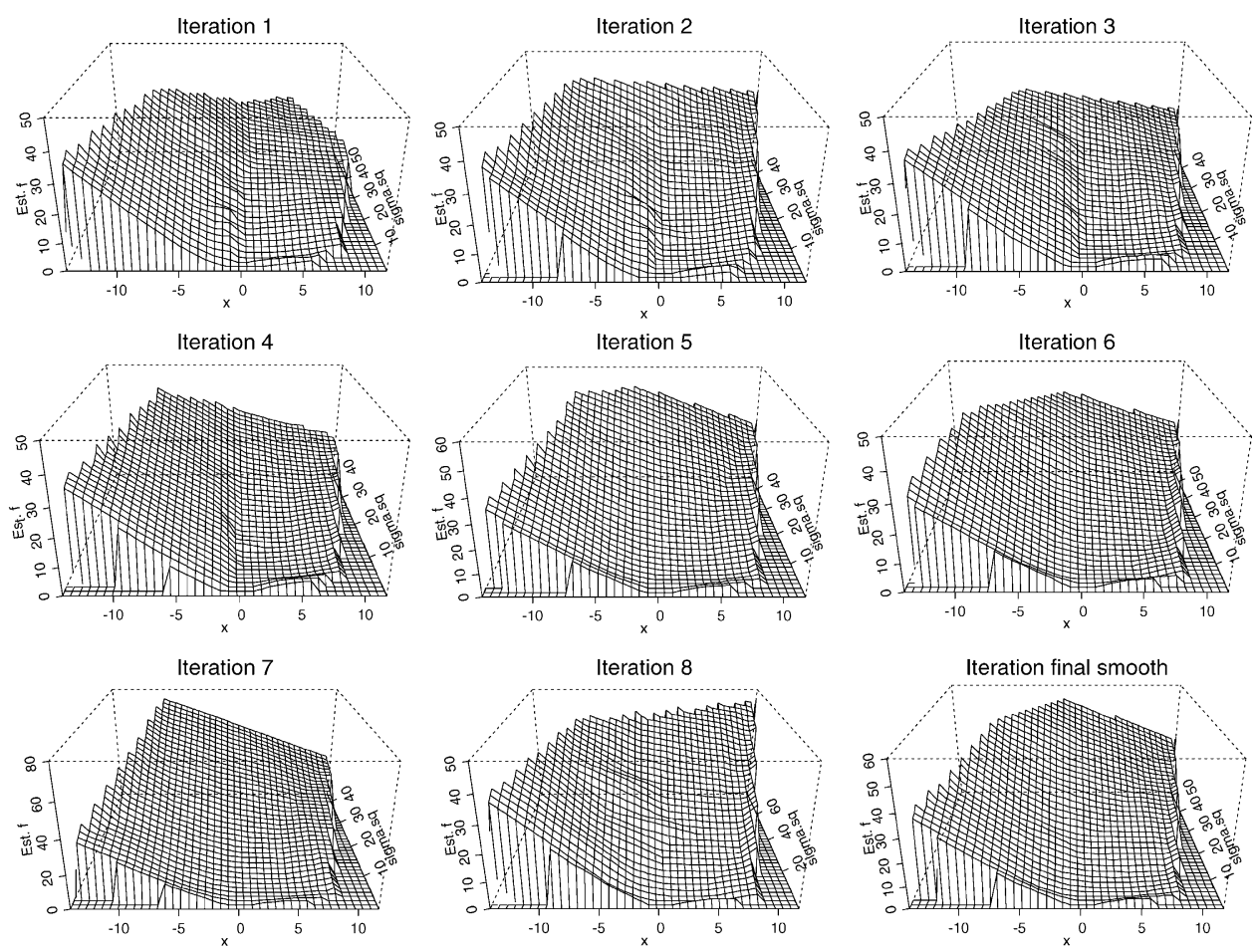

Fig. 5. Real data. The estimated surfaces $\hat{f}_{m}$ calculated at the first 8 iterations and the surface $\hat{f}$ estimated at the final smoothing stage.

predictive potential. For the nonparametric GARCH method, we evaluate AIC by using the equivalent number of degrees of freedom (Hastie and Tibshirani, 1990) in the final smoothing step of the algorithm.

From Table 5 we see that our nonparametric GARCH method is a competitive method for these data and gives results which are about as good as the full threshold model M2. The greater generality of the nonparametric method may be of great importance in other datasets where threshold models would not fit well. The gains of the nonparametric method over the $\operatorname{GARCH}(1,1)$ model amount to a $7.8 \%$ decrease of the $L_{2}$ measure and a relevant difference in AIC equal to about 20. Note that the true improvement is possibly masked by noise since $X_{t}^{2}$ is a noisy estimate of the unobservable target $\sigma_{t}^{2}$.

\section{Extensions}

The iterative idea of our estimation algorithm can be extended in a variety of ways and combined with other nonparametric modelling techniques. 

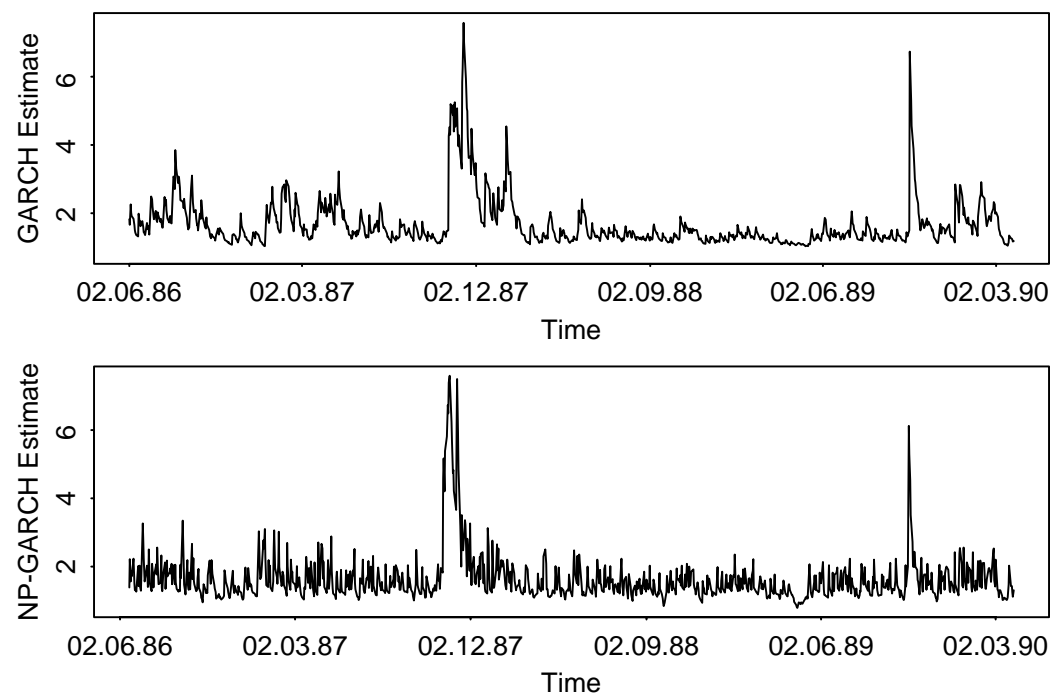

Fig. 6. Real data. A comparison of volatilities estimated by ordinary parametric $\operatorname{GARCH}(1,1)$ modelling and nonparametric estimation (after the final smooth).

Table 5

Goodness of fit measures for BMW data

\begin{tabular}{llll}
\hline Model & $L_{2}$ & - LL & AIC \\
\hline GARCH(1,1) & 106.6 & 1867.6 & 3707.9 \\
M1 & 104.2 & 1856.7 & 3687.5 \\
M2 & 96.6 & 1854.9 & 3688.6 \\
NP-GARCH & 98.3 & 1836.1 & 3688.0 \\
\hline
\end{tabular}

\subsection{Nonparametric $\mathrm{GARCH}(p, q)$}

The estimation algorithm in Section 2.1 and its justification easily extend to the nonparametric $\operatorname{GARCH}(p, q)$ model with $0 \leqslant p, q<\infty$. Eq. (1) is now generalized to

$$
\begin{aligned}
X_{t} & =\sigma_{t} Z_{t}, \\
\sigma_{t}^{2} & =f\left(X_{t-1}, \ldots, X_{t-p}, \sigma_{t-1}^{2}, \ldots, \sigma_{t-q}^{2}\right) .
\end{aligned}
$$

The $m$ th iteration step in the estimation algorithm is then

$$
\hat{\sigma}_{t, m}^{2}=\hat{f}_{m}\left(X_{t-1}, \ldots, X_{t-p}, \hat{\sigma}_{t-1, m-1}^{2}, \ldots, \hat{\sigma}_{t-q, m-1}^{2}\right),
$$

where $\hat{f}_{m}$ is based on a $p+q$-variate smoother of $X_{t}^{2}$ versus the variables $X_{t-1}, \ldots, X_{t-p}$, $\hat{\sigma}_{t-1, m-1}^{2}, \ldots, \hat{\sigma}_{t-q, m-1}^{2}$. Justification of such an extension can be given as in Section 2.2 
by replacing Assumption A1 with

$$
\begin{aligned}
& \sup _{x \in \mathbb{R}^{p}}\left|f\left(x_{1}, \ldots, x_{p}, \sigma_{1}^{2}, \ldots, \sigma_{q}^{2}\right)-f\left(x_{1}, \ldots, x_{p}, \tau_{1}^{2}, \ldots, \tau_{q}^{2}\right)\right| \leqslant \sum_{i=1}^{q} d_{i}\left|\sigma_{i}^{2}-\tau_{i}^{2}\right| \\
& \quad \text { for some } 0<d_{1}, \ldots, d_{q}<1 \quad \text { with } \sum_{i=1}^{q} d_{i}=D<1
\end{aligned}
$$

for all $\sigma^{2}, \tau^{2} \in\left(\mathbb{R}^{+}\right)^{q}$.

\subsection{Nonparametric additive GARCH}

The model in (1) or the higher-order model of Section 4.1 can be modified to the case where $\sigma_{t}^{2}$ is a general additive function of lagged values and volatilities. The estimation algorithm is simply adjusted: the smoothing operations are performed according to the additive structure of $\sigma_{t}^{2}$ by using the backfitting algorithm. For generalised additive models and their estimation see Hastie and Tibshirani (1990).

\subsection{Nonparametric ARMA-GARCH of first order}

The estimation algorithm also extends to hybrid ARMA-GARCH models; for notational simplicity we focus on the first order case. Consider

$$
\begin{aligned}
& X_{t}=\mu_{t}+\sigma_{t} Z_{t}, \\
& \mu_{t}=g\left(X_{t-1}, \mu_{t-1}\right), \\
& \sigma_{t}^{2}=f\left(\left(X_{t-1}-\mu_{t-1}\right), \sigma_{t-1}^{2}\right),
\end{aligned}
$$

where $g: \mathbb{R} \times \mathbb{R} \rightarrow \mathbb{R}$ is a nonparametric function giving the conditional mean of the process; all other elements of the model are as in (1).

Observe that

$$
\mathbb{E}\left[X_{t} \mid \mathscr{F}_{t-1}\right]=g\left(X_{t-1}, \mu_{t-1}\right)
$$

and

$$
\operatorname{Var}\left[X_{t} \mid \mathscr{F}_{t-1}\right]=f\left(\left(X_{t-1}-\mu_{t-1}\right), \sigma_{t-1}^{2}\right) .
$$

This suggests that a similar scheme can be applied to estimate both $g$ and $f$.

We start with estimates $\hat{\mu}_{t, 0}$ and $\hat{\sigma}_{t, 0}$ of $\mu_{t}$ and $\sigma_{t}$ derived by the usual parametric means. We regress $X_{t}$ against $X_{t-1}$ and $\hat{\mu}_{t-1,0}$, weighting possibly with $\hat{\sigma}_{t, 0}^{-1}$, to estimate $g$ and hence to re-estimate $\mu_{t}$ by $\hat{\mu}_{t, 1}$. We then regress $\left(X_{t}-\hat{\mu}_{t, 1}\right)^{2}$ against $\left(X_{t-1}-\hat{\mu}_{t-1,1}\right)$ and $\hat{\sigma}_{t-1,0}^{2}$, weighting possibly with $\hat{\sigma}_{t, 0}^{-2}$, to estimate $f$ and hence to re-estimate $\sigma_{t}$ by $\hat{\sigma}_{t, 1}$. We iterate this procedure to improve estimates of conditional mean and volatility. 


\section{Discussion}

In this paper, an algorithm for fitting nonparametric GARCH models of first order has been proposed and justified, and extensions to other time series models have been suggested.

The first envisaged use for the procedure in practical applications is an exploratory one: assuming the availability of a bivariate smoother (such as loess in S-Plus) it is a simple matter to implement the iterative algorithm to investigate visually the dependence of volatility on lagged values of the time series and the volatility itself.

The second envisaged use for the method is as a volatility estimator in situations where the functional relationship between volatility and the lagged series differs markedly from standard parametric $\operatorname{GARCH}(1,1)$, particularly in situations where asymmetries appear to be present. The new estimator could then also be used to compute dynamically changing measures of risk, such as value at risk and expected shortfall conditioned on the observed past; see McNeil and Frey (2000).

A further positive feature of the approach is that it is fully nonparametric in the sense that not only is the exact functional relationship between volatility and the one-lagged values of the time series and volatility left unspecified, but also no assumptions are made regarding the distributional form of the innovation distribution. (The existence of a finite fourth moment is assumed to justify the use of weighted nonparametric regression.) A number of papers containing empirical studies (McNeil and Frey, 2000; Mikosch and Stărică, 2000) have suggested that GARCH-type models with Gaussian innovations cannot capture the strong leptokurtosis of typical financial return data. Engle and González-Rivera (1991) address this problem with an alternative semiparametric approach using $\mathrm{ARCH}$ dynamics together with a general nonparametric form for the innovation distribution.

In summary, the proposed algorithm for nonparametric GARCH is an attractive flexible fitting method that requires neither the specification of the functional form of the volatility nor that of the innovation distribution.

Of course the GARCH framework is not the only way to approach volatility estimation. Another stream of models are the stochastic volatility (SV) models; see Shephard (1996). In contrast to the present model (1), the SV model has a fully latent volatility process with no observable structure. It can be formalised as a state space model

$$
\begin{aligned}
& X_{t}=\sigma_{t} Z_{t}, \\
& \left\{\sigma_{t}^{2}\right\}=\text { stationary stochastic process of a pre-specified form }
\end{aligned}
$$

with $Z_{t}$ as in (1), and $\sigma_{t}^{2}$ conditionally independent of $\left\{X_{s} ; s<t\right\}$ given $\left\{\sigma_{s}^{2} ; s<t\right\}$. Note that this conditional independence structure is not true for the nonparametric GARCH model. The volatility in (1) is only partially hidden, since the lagged value $X_{t-1}$ contributes to the partial observability of $\sigma_{t}^{2}$. The SV model in (3) cannot be fitted with the iterative procedure described in this paper. If the process $\left\{\sigma_{t}^{2}\right\}$ and the innovation distribution of $Z_{t}$ are of finite parametric form, techniques from nonlinear Kalman filtering can be used, e.g. Markov chain Monte Carlo, Gibbs sampling or recursive sampling; see for example Künsch (2000). 


\section{Appendix A}

Proof of Theorem 1. By iteration, $\sigma_{t-1, m-1}^{2} \in \mathscr{F}_{t-2}$ is independent from $\left\{Z_{s} ; s \geqslant t-1\right\}$. Since $Z_{t}$ is also independent from $\left\{X_{s} ; s<t\right\}$, we can write

$$
\begin{aligned}
\sigma_{t, m}^{2} & =\mathbb{E}\left[X_{t}^{2} \mid X_{t-1}, \sigma_{t-1, m-1}^{2}\right] \\
& =\mathbb{E}\left\{f\left(X_{t-1}, \sigma_{t-1}^{2}\right) \mid X_{t-1}, \sigma_{t-1, m-1}^{2}\right\}, m=1,2, \ldots ; t \in \mathbb{Z} .
\end{aligned}
$$

The following argument quantifies the error due to wrong starting values,

$$
\begin{aligned}
\mathbb{E}\left\{\left(\sigma_{t}^{2}-\sigma_{t, m}^{2}\right)^{2}\right\} & =\mathbb{E}\left(\left[f\left(X_{t-1}, \sigma_{t-1}^{2}\right)-\mathbb{E}\left\{f\left(X_{t-1}, \sigma_{t-1}^{2}\right) \mid X_{t-1}, \sigma_{t-1, m-1}^{2}\right\}\right]^{2}\right) \\
& \leqslant \mathbb{E}\left[\left\{f\left(X_{t-1}, \sigma_{t-1}^{2}\right)-f\left(X_{t-1}, \sigma_{t-1, m-1}^{2}\right)\right\}^{2}\right] \\
& \leqslant D^{2 m} \mathbb{E}\left[\left\{\sigma_{t-m}^{2}-\sigma_{t-m, 0}^{2}\right\}^{2}\right] .
\end{aligned}
$$

The first inequality holds since $\mathbb{E}\left\{f\left(X_{t-1}, \sigma_{t-1}^{2}\right) \mid X_{t-1}, \sigma_{t-1, m-1}^{2}\right\}$ is the best mean square error predictor of $\sigma_{t}^{2}=f\left(X_{t-1}, \sigma_{t-1}^{2}\right)$ as a function of $X_{t-1}$ and $\sigma_{t-1, m-1}^{2}$, the second inequality holds by iteration and Assumption A1. The conclusion then follows from Assumption A1, the triangle inequality for $\|\cdot\|_{2}$ and Assumption A2.

Proof of Theorem 2. Use the decomposition in (2). For the second term on the righthand side we obtain with Assumption A3 and the notation in A4,

$$
\begin{aligned}
\left\|\tilde{\sigma}_{t, m}^{2}-\sigma_{t, m}^{2}\right\|_{2} & \leqslant G\left\|\hat{\sigma}_{t-1, m-1}^{2}-\sigma_{t-1, m-1}^{2}\right\|_{2} \\
& \leqslant G\left(\Delta_{n}^{\left(\mathscr{L}_{2}\right)}+\left\|\tilde{\sigma}_{t-1, m-1}^{2}-\sigma_{t-1, m-1}^{2}\right\|_{2}\right) \\
& \leqslant \cdots \leqslant \sum_{j=1}^{m-1} G^{j} \Delta_{n}^{\left(\mathscr{L}_{2}\right)}+G^{m}\left\|\hat{\sigma}_{t-m, 0}^{2}-\sigma_{t-m, 0}^{2}\right\|_{2} .
\end{aligned}
$$

Using Theorem 1, the bound above implies

$$
\begin{aligned}
& \left\|\hat{\sigma}_{t, m}^{2}-\sigma_{t}^{2}\right\|_{2} \leqslant \sum_{j=0}^{m-1} G^{j} \Delta_{n}^{\left(\mathscr{L}_{2}\right)}+G^{m}\left\|\hat{\sigma}_{t-m, 0}^{2}-\sigma_{t-m, 0}^{2}\right\|_{2}+D^{m}\left(C_{1}+C_{2}\right), \\
& t=m+2, m+3, \ldots, n .
\end{aligned}
$$

Using the finite fourth moment of $\hat{\sigma}_{t, 0}^{2}$ and Assumption A2, this then yields the first assertion in Theorem 2. The other follows by the choice of $m_{n}$ yielding $\max \left\{D^{m_{n}}, G^{m_{n}}\right\}=$ $\mathrm{O}\left(\Delta_{n}^{\left(\mathscr{L}_{2}\right)}\right)$.

\section{References}

Bollerslev, T., 1986. Generalised autoregressive conditional heteroscedasticity. J. Econometrics 51, 307-327. Bollerslev, T., Chou, R., Kroner, K., 1992. ARCH modeling in finance. J. Econometrics 52, 5-59. 
Bollerslev, T., Engle, R., Nelson, D., 1994. ARCH models. In: Engle, R., McFadden, D. (Eds.), Handbook of Econometrics, Vol. 4. North-Holland, Amsterdam, pp. 2959-3038.

Engle, R., 1982. Autoregressive conditional heteroskedasticity with estimates of the variance of U.K. inflation. Econometrica 50, 987-1008.

Engle, R., Bollerslev, T., 1986. Modeling the persistence of conditional variances. Econometric Rev. 5, 1-50.

Engle, R., González-Rivera, G., 1991. Semiparametric ARCH models. J. Business Econom. Statist. 9, 345359.

Fornari, F., Mele, A., 1997. Signa and volatility switching ARCH models. J. Appl. Econometrics 12, 49-65.

Glosten, L., Jagannathan, R., Runkle, D., 1993. On the relation between the expected value and the volatility of the nominal excess return on stocks. J. Finance 48 (5), 1779-1801.

Hastie, T., Tibshirani, R., 1990. Generalized Models. Chapman \& Hall, New York.

Künsch, H.R., 2000. State space and hidden Markov models. In: Barndorff-Nielsen, O., Cox, D., Klüppelberg, C. (Eds.), Complex Stochastic Systems. Monographs on Statistics and Applied Probability 87. Chapman \& Hall/CRC Press LLC.

McNeil, A., Frey, R., 2000. Estimation of tail-related risk measures for heteroscedastic financial time series: an extreme value approach J. Empirical Finance 7, 271-300.

Mikosch, T., Stărică, C., 2000. Limit theory for the sample autocorrelations and extremes of a $\operatorname{GARCH}(1,1)$ process. Ann. Statist. 28, 1427-1451.

Nelson, D.B., 1991. Conditional heteroscedasticity in asset pricing: a new approach. Econometrica 59, $347-$ 370.

Shephard, N., 1996. Statistical aspects of arch and stochastic volatility. In: Cox, D., Hinkley, D., Barndorff-Nielsen, O. (Eds.), Time Series Models in Econometrics, Finance and other Fields. Chapman \& Hall, London, pp. 1-55.

Stone, C.J., 1982. Optimal global rates of convergence for nonparametric regression. Ann. Statist. 10, 1040 1053.

Yang, L., Härdle, W., Nielsen, J.P., 1999. Nonparametric autoregression with multiplicative volatility and additive mean. J. Time Ser. Anal. 20, 579-604. 